\title{
Objeção de Consciência e Aborto Legal: Atitudes de Estudantes de Medicina
}

\author{
Conscientious Objection and Legal \\ Abortion: Medical Students' Attitudes
}

\author{
Alberto Madeiro \\ Andréa Rufino ${ }^{I}$ \\ Paloma Santos ${ }^{I}$ \\ Geisa Bandeiral \\ Isadora Freitas ${ }^{l}$
}

\section{PALAVRAS-CHAVE}

- Aborto Legal;

- Estudantes de Medicina;

- Conhecimentos, Atitudes e Práticas em Saúde;

- Educação Médica.

\section{KEYWORDS}

- Legal Abortion;

- Medical Students;

- Health Knowledge, Attitudes and Practice;

- Medical Education.
Reencaminhado em: 29/08/2015

Aprovado em: 29/08/2015

\begin{abstract}
Introduction: In Brazil abortion is permitted when the woman's life is at risk, the pregnancy resulted from rape or in the case of fetal anencephaly. Objective: The aim of this study was to evaluate the attitudes of medical students towards conscientious objection to abortion. Methods: Medical students from Piaui were asked to answer an anonymous electronic questionnaire with questions about sociodemographic characteristics, conscientious objection to abortion and ethical obligations in the case of objection. Results: The answer rate was $66.7 \%(n=1,174)$. Whereas $13.2 \%$ of the students would have presented conscientious objection in the case of any threat to the woman's life, 31.6\% would have objected in the case of fetal anencephaly, and $50.8 \%$ in the case of rape. Among the students who would object to abortion in the case of rape, 54\% would not refer the woman to another professional and $72.5 \%$ would not explain the available treatment options. Religion was the only characteristic associated with refusal. Conclusions: Conscientious objection to abortion in the case of rape was more frequent than other circumstances foreseen by Brazilian legislation. Students with religious affiliations were more associated with refusal.
\end{abstract}




\section{INTRODUÇÃO}

O aborto é permitido somente em algumas circunstâncias no Brasil. De acordo com o Código Penal de 1940, ele pode ser autorizado quando a gravidez é decorrente de estupro ou quando há risco de morte da mulher1. Em 2012, o Supremo Tribunal Federal incorporou a gravidez de feto com anencefalia como possibilidade de interrupção legal da gestação ${ }^{2}$. O atendimento das mulheres nas situações previstas em lei é feito em unidades de referência públicas conhecidas como "serviços de aborto legal"3. Atualmente, existem 65 serviços de referência para o aborto legal no País, a maioria localizada nas grandes cidades brasileiras ${ }^{4}$.

O Ministério da Saúde estabeleceu diretrizes para o atendimento de mulheres vítimas de violência sexual e o aborto previsto em lei. Essas diretrizes normatizam que os médicos têm o direito individual de objeção de consciência à prática do aborto. A recusa de consciência não será aceita, porém, se não houver outro médico para atender a mulher, se houver risco de morte ou se a omissão do atendimento puder causar danos $^{5}$. No caso brasileiro, a recusa de consciência acionada por médicos pode representar uma barreira para o atendimento das mulheres, uma vez que há dependência dos serviços públicos para a interrupção segura da gestação e, em muitas cidades, existe somente um serviço disponível.

Quando alega objeção de consciência, o médico deve justificar sua recusa, informar as possibilidades de tratamento e encaminhar a mulher a outro profissional ${ }^{6,7}$. Inquéritos realizados em outros países demonstraram que são os médicos do sexo masculino e com filiação religiosa os que mais frequentemente se recusam a justificar a objeção e encaminhar a paciente à assistência em saúde ${ }^{8,9}$. O Código de Ética Médica brasileiro garante ao médico o direito de objeção, mas impõe a ele a responsabilidade de não dificultar o acesso ao aborto previsto em lei ${ }^{10}$.

As atitudes de estudantes em relação ao aborto variam dependendo das circunstâncias da gravidez. Em um levantamento realizado em 2008 entre estudantes de Medicina do Reino Unido, 19,8\% afirmaram que não fariam o aborto em menor de idade vítima de estupro, 23,5\% não fariam em caso de falha contraceptiva e 29,5\% não fariam em feto com malformação ${ }^{11}$. Dados diferentes foram obtidos entre estudantes da África do Sul: 59,5\% disseram que teriam disponibilidade para participar do aborto em caso de severa malformação fetal ${ }^{12}$.

Não existem dados sobre objeção de consciência ao aborto entre médicos e estudantes de Medicina no Brasil. A compreensão das atitudes dos estudantes de Medicina sobre o aborto pode permitir uma previsão do comportamento futuro dos médicos sobre o tema. Este artigo descreve as atitudes de estudantes de Medicina brasileiros em relação à objeção de consciência ao aborto. Nós perguntamos aos estudantes de Medicina se eles se recusariam a participar das três possibilidades de aborto permitidas pela lei brasileira e se, em caso de objeção, justificariam a recusa, explicariam todas as opções de tratamento às mulheres e as encaminhariam a outro profissional. Nosso objetivo secundário foi determinar se a idade e o sexo dos estudantes, o ano em que se encontravam no curso médico e a prática de religião influenciavam a objeção de consciência e as responsabilidades éticas após a recusa.

\section{METODOLOGIA}

Desenho do estudo

Realizou-se estudo descritivo e transversal com os estudantes de Medicina da Universidade Federal do Piauí, da Universidade Estadual do Piauí, do Centro Universitário Novafapi e da Faculdade Integral Diferencial, localizados na cidade de Teresina, Piauí. Todos os 1.760 alunos de todos os anos do curso médico (pré-clínico e clínico) foram contactados por e-mail institucional e convidados a participar do estudo após autorização formal das escolas médicas. A amostra foi de conveniência, uma vez que as escolas não forneceram listas de estudantes por sexo, idade ou ano do curso médico. Foram enviados convites de $1^{\text {o }}$ de setembro a 31 de dezembro de 2012. Dois lembretes mensais da pesquisa foram reenviados. O Comitê de Ética em Pesquisa da Universidade Estadual do Piauí aprovou o desenho do estudo quanto aos seus aspectos éticos (CAAE 02896312.9.0000.5209). O Termo de Consentimento Livre e Esclarecido foi obtido eletronicamente de todos os estudantes.

\section{Coleta de dados}

Um questionário estruturado anônimo e eletrônico foi disponibilizado em website específico para o estudo. O instrumento de pesquisa foi composto por três seções, com dez questões fechadas. A seção A era composta por quatro questões sobre idade, sexo, ano do curso médico e religião. A seção B perguntou sobre objeção de consciência nas circunstâncias em que o aborto é permitido no País, com três questões avaliando se o estudante recusaria a realização do aborto em caso de estupro, de anencefalia e de risco de morte da mulher. Na seção C, foi questionado se os estudantes, em caso de objeção de consciência ao aborto, justificariam a recusa, explicariam à mulher todas as opções de tratamento e a encaminhariam a outro colega que não recusasse o atendimento. As categorias de respostas para as seções B e C foram "sim" ou "não". O questionário foi desenhado levando em consideração estudos prévios sobre atitudes e opiniões sobre o aborto entre médicos e estudantes de Medicina ${ }^{8,11,13}$.

Um pré-teste do questionário foi feito com 30 estudantes (cinco para cada ano do curso médico), para avaliação do en- 


\begin{tabular}{|c|c|c|}
\hline \multicolumn{3}{|c|}{$\begin{array}{c}\text { TABELA } 1 . \\
\text { Dados demográficos dos estudantes de Medicina. } \\
\text { Teresina, Piauí, } 2012 .\end{array}$} \\
\hline Variáveis & $\mathbf{n}$ & $\%$ \\
\hline \multicolumn{3}{|l|}{ Faixa etária (anos) } \\
\hline $17-20$ & 329 & 28,0 \\
\hline $21-25$ & 795 & 67,7 \\
\hline$>25$ & 50 & 4,3 \\
\hline \multicolumn{3}{|l|}{ Sexo } \\
\hline Feminino & 566 & 48,2 \\
\hline Masculino & 608 & 51,8 \\
\hline \multicolumn{3}{|l|}{ Ano do curso } \\
\hline $1^{\circ}-2^{\circ}$ (pré-clínico) & 409 & 34,8 \\
\hline $3^{3}-4^{\circ}$ (clínico) & 405 & 34,5 \\
\hline $5^{\circ}-6^{\circ}$ (internato) & 360 & 30,7 \\
\hline \multicolumn{3}{|l|}{ Religião } \\
\hline Não & 218 & 18,6 \\
\hline Sim & 956 & 81,4 \\
\hline Católica & 819 & 69,8 \\
\hline Evangélica & 87 & 7,4 \\
\hline Outras & 50 & 4,3 \\
\hline
\end{tabular}

tendimento das perguntas, do tempo gasto na resposta e da adoção adequada de termos técnicos. Somente após a reformulação desencadeada pelas sugestões recebidas, o questionário foi disponibilizado eletronicamente. Todas as respostas obtidas dos estudantes no pré-teste foram descartadas.

\section{Análise dos dados}

Os dados foram tabulados em planilha do Excel 2007 e descritos sob a forma de frequências e percentuais. A análise estatística bivariada foi realizada utilizando-se o programa SPSS versão 18.0 (Inc., Chicago IL). Para determinar se havia associação entre as características demográficas e a objeção de consciência ao aborto, foi empregado o teste qui-quadrado de Pearson. O nível de significância adotado foi de 5\%.

\section{RESULTADOS}

Taxa de resposta e dados demográficos

Houve obtenção de questionários completos de 1.174 estudantes, com taxa de resposta de $66,7 \%$. A Tabela 1 mostra os dados demográficos dos estudantes. A idade variou de 17 a 39 anos (dado não mostrado na tabela), com predomínio da faixa de 21 a 25 anos $(67,7 \%)$. Pouco mais da metade $(51,8 \%)$ dos respondentes era do sexo masculino. A distribuição dos alunos pelos anos de curso médico foi semelhante, com pequena preponderância de estudantes nos anos pré-clínicos do curso (34,8\%). Sobre a declaração de filiação religiosa, 18,6\% informaram não ter religião e 69,8\% eram católicos.

\section{Objeção de consciência ao aborto}

A declaração de objeção de consciência pelos estudantes variou de acordo com as circunstâncias do aborto permitidas pela legislação brasileira (Tabela 2). Enquanto 13,2\% dos estu-

TABela 2.

Objeção de consciência dos estudantes de Medicina ao aborto legal de acordo com as variáveis demográficas. Teresina, Piauí, 2012.

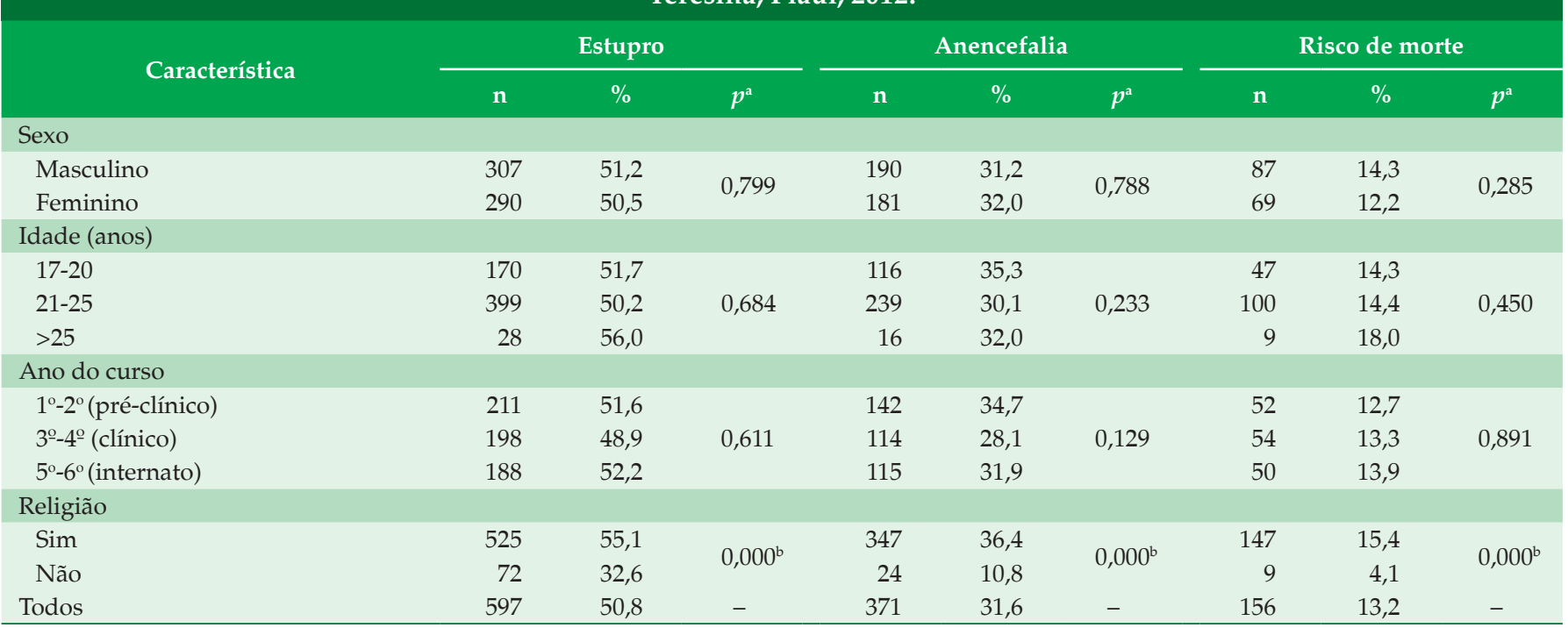

${ }^{a} \mathrm{O}$ valor de p reflete a análise bivariada entre características demográficas e objeção de consciência.

${ }^{b}$ Estatisticamente significante $(p<0,05)$. 


\begin{tabular}{|c|c|c|c|c|c|c|c|c|c|}
\hline \multicolumn{10}{|c|}{$\begin{array}{l}\text { Responsabilidades éticas envolvidas na objeção de consciência ao aborto de } \\
\text { acordo com as variáveis demográficas.Teresina, Piauí, } 2012\end{array}$} \\
\hline \multirow{3}{*}{ Característica } & \multicolumn{3}{|c|}{ Justificaria a objeção } & \multicolumn{3}{|c|}{ Explicaria todas as opções } & \multicolumn{3}{|c|}{ Encaminharia a outro colega } \\
\hline & \multicolumn{3}{|c|}{ Não } & \multicolumn{3}{|c|}{ Não } & \multicolumn{3}{|c|}{ Não } \\
\hline & $\mathbf{n}$ & $\%$ & $p^{\mathrm{a}}$ & $\mathrm{n}$ & $\%$ & $p^{\mathrm{a}}$ & n & $\%$ & $p^{\mathrm{a}}$ \\
\hline Risco de morte $(n=156)$ & 9 & 5,8 & - & 27 & 17,3 & - & 33 & 21,1 & - \\
\hline Anencefalia $(\mathrm{n}=371)$ & 24 & 6,4 & - & 117 & 31,5 & - & 109 & 29,4 & - \\
\hline Estupro (n = 597) & 42 & 7,0 & - & 433 & 72,5 & - & 322 & 54,0 & - \\
\hline \multicolumn{10}{|l|}{ Sexo } \\
\hline Feminino & 30 & 5,3 & \multirow{2}{*}{0,583} & 225 & 39,8 & \multirow{2}{*}{0,537} & 180 & 31,8 & \multirow{2}{*}{0,053} \\
\hline Masculino & 28 & 4,8 & & 231 & 38,0 & & 149 & 24,5 & \\
\hline \multicolumn{10}{|l|}{ Idade (anos) } \\
\hline $17-20$ & 11 & 3,3 & \multirow{3}{*}{0,252} & 130 & 39,5 & \multirow{3}{*}{0,840} & 98 & 29,8 & \multirow{3}{*}{0,640} \\
\hline $21-25$ & 45 & 5,7 & & 364 & 38,4 & & 216 & 27,2 & \\
\hline$>25$ & 2 & 4,0 & & 21 & 42,0 & & 15 & 30,0 & \\
\hline \multicolumn{10}{|l|}{ Anos do curso } \\
\hline $1^{\circ}-2^{\circ}$ & 16 & 3,9 & \multirow{3}{*}{0,056} & 155 & 37,9 & \multirow{3}{*}{0,214} & 125 & 30,6 & \multirow{3}{*}{0,160} \\
\hline $3^{\circ}-4^{\circ}$ & 16 & 3,9 & & 148 & 36,5 & & 100 & 24,7 & \\
\hline $5^{\mathrm{o}-}-6^{\mathrm{o}}$ & 26 & 7,2 & & 153 & 42,5 & & 104 & 28,9 & \\
\hline \multicolumn{10}{|l|}{ Religião } \\
\hline Sim & 53 & 5,5 & \multirow{2}{*}{$0,046^{\mathrm{b}}$} & 389 & 40,7 & \multirow{2}{*}{$0,006^{\mathrm{b}}$} & 292 & 30,5 & \multirow{2}{*}{$0,000^{\mathrm{b}}$} \\
\hline Não & 5 & 2,3 & & 67 & 30,7 & & 37 & 17,0 & \\
\hline Todos $(\mathrm{n}=1.174)$ & 58 & 4,9 & - & 456 & 38,8 & - & 329 & 28,0 & - \\
\hline
\end{tabular}

${ }^{a} \mathrm{O}$ valor de $p$ reflete a análise bivariada entre características demográficas e as responsabilidades éticas.

${ }^{b}$ Estatisticamente significante $(p<0,05)$.

dantes informaram que recusariam realizar o aborto em caso de risco de morte da mulher, $31,6 \%$ disseram que objetariam quando houvesse anencefalia fetal, e 50,8\%, em caso de estupro. Nas três situações (risco de morte, anencefalia e estupro), os estudantes que tinham religião estiveram mais associados à objeção de consciência do que os estudantes que não tinham religião $\left(\chi^{2}=21,4, p<0,000 ; \chi^{2}=54,8, p<0,000 ; \chi^{2}=37,6\right.$, $\mathrm{p}<0,000$, respectivamente). Não houve associação significativa da objeção com sexo, idade e ano do curso.

Responsabilidades éticas envolvidas com a objeção de consciência ao aborto

A Tabela 3 mostra as atitudes dos estudantes perante as obrigações éticas envolvidas com a declaração de objeção de consciência. Entre os estudantes, apenas uma minoria (4,9\%) não justificaria a objeção para a paciente. Quase um terço (28\%) não encaminharia a mulher a outro médico que não tivesse objeção e 38,8\% não detalhariam para ela todas as opções possíveis para a realização do aborto. As respostas somente dos estudantes que declararam objeção de consciência em caso de estupro, no entanto, demonstram que 54\% não encaminha- riam a mulher a outro profissional e 72,5\% não explicariam a ela todas as opções de tratamento. Não houve associação de sexo, idade e ano do curso com as atitudes. Por sua vez, estudantes com religião, quando comparados àqueles sem religião, mostraram associação significativa com não justificar a recusa $\left(\chi^{2}=3,993, p=0,046\right)$, não explicar todas as opções $\left(\chi^{2}=\right.$ $7,408, p<0,001)$ e não encaminhar a mulher a outro profissio$\operatorname{nal}\left(\chi^{2}=17,584, p<0,001\right)$.

\section{DISCUSSÃO}

O aborto é um dos tópicos que mais provocam controvérsia moral no campo da saúde reprodutiva. Nas duas últimas décadas, com o aumento da proteção dos direitos sexuais e reprodutivos em todo o mundo, também houve maior frequência de objeção de consciência, principalmente em países onde a influência da religião é maior. Assim, a oferta de aborto seguro pode ser dificultada pela objeção de consciência alegada por médicos. Não existem dados sobre objeção de consciência entre médicos brasileiros. No entanto, como em muitos estados há somente um serviço de referência, na prática, quando um ou mais médicos recusam a realização do aborto, isso 
pode significar que a mulher enfrentará obstáculos para ter sua solicitação atendida. Nossa expectativa é que, por meio da observação das atitudes de estudantes de Medicina, se possa desenhar um panorama aproximado de sua futura atuação profissional na comunidade.

Observamos que os estudantes de Medicina apresentaram objeção de consciência diferenciada nas três possibilidades de aborto previstas em lei no Brasil. Chama a atenção o fato de que os estudantes são menos propensos a apresentar objeção quando há uma razão médica que o justifique, como no caso da anencefalia e de risco de morte da mulher. Por outro lado, metade dos alunos (50,8\%) parece não considerar o estupro como fator moralmente relevante para a interrupção da gravidez. Outras pesquisas, realizadas em países com legislação mais liberal do que a brasileira, demonstraram que a intenção dos estudantes em participar do aborto é influenciada pelas circunstâncias da gravidez. No Reino Unido, um levantamento em 2008 mostrou que $67 \%$ dos estudantes fariam o aborto em caso de risco de morte da mulher, 59\% fariam em caso de gravidez por estupro e apenas $37 \%$ o fariam em caso de gravidez indesejada ${ }^{13}$. Dados diferentes foram obtidos entre estudantes da Espanha, entre os quais 86\% disseram que teriam disponibilidade para participar do aborto por estupro $^{14}$.

Entre os estudantes que manifestaram recusa, houve associação significativa com a filiação religiosa. A religião é um bem conhecido fator que influencia a atitude em relação ao aborto. No entanto, em um país laico, como o Brasil, as razões e crenças religiosas não devem ser definidoras para o estabelecimento das políticas públicas e da assistência em saúde. Médicos com filiação religiosa apresentam menor disponibilidade para aconselhar e participar de procedimentos no aborto eletivo $^{8,15}$. Pesquisa conduzida em quatro universidades do Reino Unido mostrou que $76,2 \%$ dos alunos muçulmanos entrevistados acreditam que médicos têm o direito de recusar a participação em qualquer procedimento, inclusive o aborto $^{11}$. Apesar de o questionário não contemplar as razões para a recusa, na maior parte das vezes os argumentos de ordem religiosa para tornar o aborto inaceitável se fundamentam na crença da sacralidade da vida humana e da existência de uma pessoa desde a fecundação ${ }^{15}$.

Ao contrário do verificado em outras pesquisas, não observamos associações de sexo, idade e ano do curso com a recusa em participar do aborto. Há evidências de que estudantes da fase pré-clínica apresentam menor aceitação do aborto que aqueles de anos mais adiantados ${ }^{16}$. Parece que a oferta de conteúdos sobre aborto e outros temas de planejamento familiar durante a graduação, sob a forma tanto de discussão ética como de treinamento prático, pode influenciar as atitudes e a intenção de trabalhar com aborto no futuro ${ }^{17,18}$. Não se conhece como o tema aborto é abordado nas escolas médicas brasileiras, mas um estudo em 2005 nos Estados Unidos mostrou que $17 \%$ das escolas médicas naquele país não oferecem nenhum conteúdo ${ }^{19}$. Mesmo naquelas onde esse tema faz parte do currículo, $67 \%$ dedicam menos de duas horas à sua discussão ${ }^{20}$.

Nossos dados mostraram que a maioria dos estudantes informou que seria eticamente aceitável justificar a objeção de consciência em caso de aborto. Porém, quase 40\% deles julgaram que não era obrigação revelar todas as opções para o tratamento e $28 \%$ não encaminhariam a mulher a outro profissional. É importante destacar que essas atitudes foram ainda menos encontradas entre aqueles que recusariam o aborto em caso de estupro, uma vez que três em cada quatro desses estudantes não encaminhariam a mulher a outro atendimento. Esses dados divergem dos encontrados em estudos de outros países. Na África do Sul, somente 12,5\% dos estudantes se recusariam a realizar o aborto e encaminhar a mulher ${ }^{12}$, ao passo que nos Estados Unidos, embora 30\% dos alunos fossem contra o aborto, somente 3\% afirmaram que não encaminhariam a paciente a outro profissional ${ }^{21}$

Para alguns médicos, o fato de encaminhar a paciente a outro profissional que não objete o aborto pode ser considerado cumplicidade, quase comparável à prática do procedimento. Para que isso não aconteça, deve haver distinção entre as crenças pessoais e os deveres profissionais. Os médicos e estudantes de Medicina que defendem seu direito de se opor a um tratamento por questões religiosas ou morais devem ter a clareza de suas responsabilidades para garantir ou, pelo menos, não obstruir o acesso ao tratamento recusado. Quando houver objeção, os médicos deverão explicar as razões de sua objeção, fornecer todas as opções de tratamento e encaminhar a paciente a outro profissional que não recuse. O dever de proporcionar benefícios e prevenir danos à saúde antecede a objeção de consciência, e, se essas exigências éticas não forem cumpridas, a obrigação de cuidado médico estará ameaçada.

Este estudo apresenta algumas limitações. Primeiro, a amostra de conveniência pode ter causado viés de seleção, o que impede a generalização dos dados. Segundo, os estudantes podem mudar de opinião com o passar do tempo, principalmente levando em consideração experiências profissionais no futuro. Terceiro, os dados referem-se a um estudo local. As atitudes dos estudantes neste estudo podem não ser semelhantes àquelas de alunos de outros países ou mesmo de outras regiões do Brasil, com contextos religioso, educacional e cultural diversos. 


\section{CONCLUSÕES}

Apesar das limitações, este é o primeiro estudo realizado no Brasil entre estudantes de Medicina sobre a objeção de consciência ao aborto. O principal achado deste estudo demonstra que pouco mais da metade dos estudantes entrevistados, principalmente os religiosos, apresenta recusa de atendimento em aborto em caso de estupro. A oferta de aborto seguro necessita da capacitação de médicos com conhecimentos, habilidades e atitudes adequadas para garantir o acesso das mulheres a esse direito em saúde. Não há como prever o impacto desses resultados na oferta de aborto no Brasil; porém, se as atitudes desses estudantes forem reproduzidas nos futuros profissionais médicos, é possível que a objeção de consciência represente uma barreira para mulheres vítimas de estupro que solicitam a interrupção da gravidez.

Independentemente de suas opiniões, estudantes de Medicina e profissionais de saúde devem compreender suas responsabilidades quando declaram objeção a um procedimento médico que, embora controverso, é permitido por lei. Nenhum estudante de Medicina ou médico é obrigado a realizar um procedimento com o qual não concorda, mas, ao mesmo tempo, aquele que alega objeção de consciência deve estar preparado para facilitar e garantir o acesso ao cuidado em saúde recusado. A explicação isenta das opções possíveis para o tratamento recusado é uma aposta na capacidade de decisão autônoma da mulher e um abandono do paternalismo médico como forma de atuação profissional. Sendo a objeção de consciência uma questão individual do médico, as mulheres não têm que ter ônus por ela. Mesmo que a recusa aconteça em caso de aborto, os estudantes de Medicina precisam ser treinados para compreender que o profissionalismo exige a oferta da melhor informação, o encaminhamento adequado e a realização do tratamento garantido pela lei. A oferta de conhecimentos e a capacitação para a assistência ao aborto legal devem fazer parte do currículo das escolas médicas como estratégia para sensibilizar os futuros médicos para o atendimento integral à saúde reprodutiva das mulheres.

\section{REFERÊNCIAS}

1. Brasil. Ministério da Justiça. Código Penal Brasileiro. Decreto-Lei $\mathrm{n}^{\circ}$ 2.848, de 7 de dezembro de 1940. Artigo 128. Disponível em: http://www.planalto.gov.br/ccivil_03/ decreto-lei/Del2848.htm. Acesso em: 21 abr. 2013.

2. Brasil. Supremo Tribunal Federal. Arguição de Descumprimento de Preceito Fundamental n ${ }^{\circ} 54$. Diário da Justiça Eletrônico no 78/2012, de 20/04/2012. Disponível em: http://www.stf.jus.br/portal/diarioJustica/verDiarioProcesso.asp?numDj=77\&dataPublicacaoDj=20/04/2012 \&incidente $=2226954 \&$ codCapitulo $=2 \&$ numMateria $=10 \& c$ odMateria=4. Acesso em: 21 abr. 2013.

3. Diniz D. Objeção de consciência e aborto: direitos e deveres dos médicos na saúde pública. Rev SaúdePública 2011; 45(5):981-5.

4. Diniz S. Materno-infantilism, feminism and maternal health policy in Brazil. Reprod Health Matters 2012; 20(39):125132.

5. Brasil. Ministério da Saúde. Norma Técnica: Prevenção e tratamento dos agravos resultantes da violência sexual contra mulheres e adolescentes. 2.ed. Brasília; 2005.

6. Savulescu J. Conscientious objection in medicine. BMJ 2006; 332(7536): 294-297.

7. Wicclair MR. Is conscientious objection incompatible with a physician's professional obligations? Theor Med Bioeth 2008; 29(3):171-185.

8. Curlin FA, Lawrence RE, Chin MH, Lantos JD. Religion, conscience, and controversial clinical practices. NEJM 2007; 356(6):593-600.

9. Rasinski KA, Yoon JD, Kalad YG, Curlin FA. Obstetrician-gynaecologists' opinions about conscientious refusal of a request for abortion: results from a national vignette experiment. J MedEthics 2011;37(12): 711-712.

10. Conselho Federal de Medicina. Código de Ética Médica [internet]. Brasília; 2009 [capturado em: 7 ago. 2013]. Disponível em: http://www.cremers.org.br/pdf/codigodeetica/codigo_etica.pdf.

11. Strickland SL. Conscientious objection in medical students: a questionnaire survey. J Med Ethics 2012; 38(1):22-25.

12. Buga GAB. Attitudes of medical students to induced abortion. East Afr Med J 2002; 79(5):259-62.

13. Gleeson R, Forde E, Bates E, Powell S, Eadon-Jones E, Draper H. Medical students' attitudes towards abortion: a UK survey. J MedEthics 2008; 34 (11):783-87.

14. Rodríguez-Calvo MS, Martínez-Silva IM, Soto JL, Concheiro L, Muñoz-Barús JI. University students' attitudes towards voluntary interruption of pregnancy. Legal Medicine 2012;14(4):209-13.

15. Harris LA, Cooper A, Rasinski KA, Curlin FA, Lyerly AD. Obstetrician-gynecologists' objections to and willingness to help patients obtain an abortion. ObstetGynecol 2011; 118(4):905-12.

16. Wheeler SA, Zullig LL, Reeve BB, Buga GA, Morroni C. Attitudes and intentions regarding abortion provision among medical school students in South Africa. Int J Perspect Sex Reprod Health 2012; 38 (3):154-63.

17. Shotorbani S, Zimmerman FJ, Bell JF, Ward D, Assefi N. Attitudes and intentions of future health care providers to- 
ward abortion provision. Perspec Sex Reprod Health 2004; 36(2):58-63.

18. Pace L, Sandahl Y, Backus L, Silveira M, Steinauer J. Medical Students for Choice's Reproductive Health Externships: impact on medical students' knowledge, attitudes and intentions to provide abortions. Contraception 2008; 78 (1): 31-5.

19. Espey E, Ogburn T, Chaves A, Qualls C, Leyba M. Abortion education in medical schools: a national survey. Am J ObstetGynecol 2005; 192(2):640-643.

20. Steinauer J, Rowh M, Backus L, Sandahl Y, Foster A. First impressions: what are preclinical medical students in the US and Canada learning about sexual and reproductive health? Contraception 2009; 80(1):74-80.

21. Rosenblatt RA, Robinson KB, Larson EH, Dobie SA. Medical students' attitudes toward abortion and other reproductive health services. Family Medicine 1999; 31(3):195-99.

\section{CONFLITO DE INTERESSES}

Os autores declaram não haver conflito de interesses.

\section{CONTRIBUIÇÃO DOS AUTORES}

Todos os autores participaram da confecção do projeto, da coleta e da análise dos dados, e da redação do artigo. Todos os autores revisaram os argumentos finais.

\section{ENDEREÇO PARA CORRESPONDÊNCIA}

Alberto Pereira Madeiro

Rua Olavo Bilac, 2335

Centro/Sul - Teresina

CEP 64001-120 - Piauí

E-mail: madeiro@uol.com.br 